\title{
Dynamics of Tuberculosis: The effect of Direct Observation Therapy Strategy (DOTS) in Nigeria
}

\author{
D. Okuonghae ${ }^{a 1}$ and A. Korobeinikov ${ }^{b}$ \\ ${ }^{a}$ Department of Mathematics, University of Benin, PMB 1154, Benin City, Edo State, Nigeria \\ ${ }^{b}$ MACSI, Department of Mathematics and Statistics, University of Limerick, Ireland
}

\begin{abstract}
This paper presents mathematical models for tuberculosis and its dynamics under the implementation of the direct observation therapy strategy (DOTS) in Nigeria. The models establish conditions for the eradication of tuberculosis in Nigeria based on the fraction of detected infectious individuals placed under DOTS for treatment. Both numerical and qualitative analysis of the models were carried out and the effect of the fraction of detected cases of active TB on the various epidemiological classes is investigated. The results showed that, provided that the fraction of detected infectious individuals exceeded a critical value, there exists a globally stable disease free equilibrium. However, if this critical detection level is not reached, the disease-free equilibrium will be unstable even with the very high probability successful treatment under DOTS. The results showed that DOTS expansion in Nigeria must include significant increase in the detection rate of infectious individuals; otherwise the effect in reducing the incidence in Nigeria will not be achieved disregarding the tremendous efforts in any other direction, and the huge number of undetected cases will make DOTS insignificant with respect to tuberculosis control.
\end{abstract}

Key words: tuberculosis, mathematical model, DOTS, prevention and control, case detection, global stability, basic reproduction number

AMS subject classification: 92D30, 34D23

\section{Introduction}

Tuberculosis continues to cause a high mortality in humans, especially in developing countries, with Sub-Saharan Africa having the highest per capita level of incidence [20, 22]. In 1993, the

\footnotetext{
${ }^{1}$ Corresponding author. E-mail: danny.okuonghae@corpus-christi.oxon.org
} 
World Health Organization (WHO) declared TB as a global emergency because of the rising deaths and infection rates. It is estimated that a third of the worlds population is infected with Mycobacterium tuberculosis; of the 1.7 billion people estimated to be infected with TB, 1.3 billion live in developing countries [29]. Tuberculosis is responsible for 8 to 12 million active cases and for 3 million deaths $[20,26]$.

A global control strategy adopted by the WHO to help reduce the number of active TB cases as well as promote proper treatment of patients with tuberculosis is the Direct Observation Therapy Strategy (DOTS). Non-adherence to treatment of TB results in resurgence of resistance strains, making it even more difficult to cure. DOTS have evolved into a strategy that makes it compulsory for patients to complete their treatment. The DOTS program uses a nurse or surrogate who deliver and supervise the patients taking all the doses of their drugs rather than relying on the patients to take the drugs on their own [10]. The patients may choose either to come to a health facility (the clinic based DOT) [10,13], or to be visited wherever they are, e.g. at work, home or shelter (the community based DOT) [11].

DOTS seems to be highly effective in promoting successful treatment: a comparison of the self-treatment versus various forms of DOTS has shown that the successful completion of the treatment is a significantly more likely outcome when the treatment is supervised $[10,12]$. Although implementation of a program such as DOTS is essential for reducing TB relapse and preventing emergence of drug-resistant strains, its impact on the spread and the control of tuberculosis is not clear $[2,4]$.

Nigeria has been ranked fourth among the 22 countries designated by the WHO as the highburden countries (HBC) for TB. Nigeria is also said to have the highest number of new TB cases in Africa [23, 33] having about 300,000 of estimated TB cases recorded each year, which result in 30,000 deaths annually. Moreover, the total of the notified cases for TB of all forms increased from 46,473 in 2003 to 59, 493 in 2004.

With introduction of DOTS in Nigeria, the situation has not improved significantly as detection rate remains at a low $21 \%$ while treatment rate is $59 \%$ in 2003 , which are the lowest levels among the high burden countries (HBC) [33]. Figure 1 shows DOTS implementation and surveillance in Nigeria. What is happening with the undetected cases of active TB? How are these affect the dynamics of TB in Nigeria? What are the conditions for the detection rate (the proportion of the detected cases treated under a DOTS program) that can ensure the eradication of TB, or at least minimize the incidence? Are the undetected cases undermining the efforts of the DOTS with respect to reducing the incidence of TB in Nigeria and effecting proper and efficient treatment policy for patients with active tuberculosis?

In this paper, to explore the overall effect of the DOTS on the dynamics of tuberculosis in Nigeria, we formulate a mathematical model taking into cognisance the fraction of detected cases undergoing treatment under DOTS. 


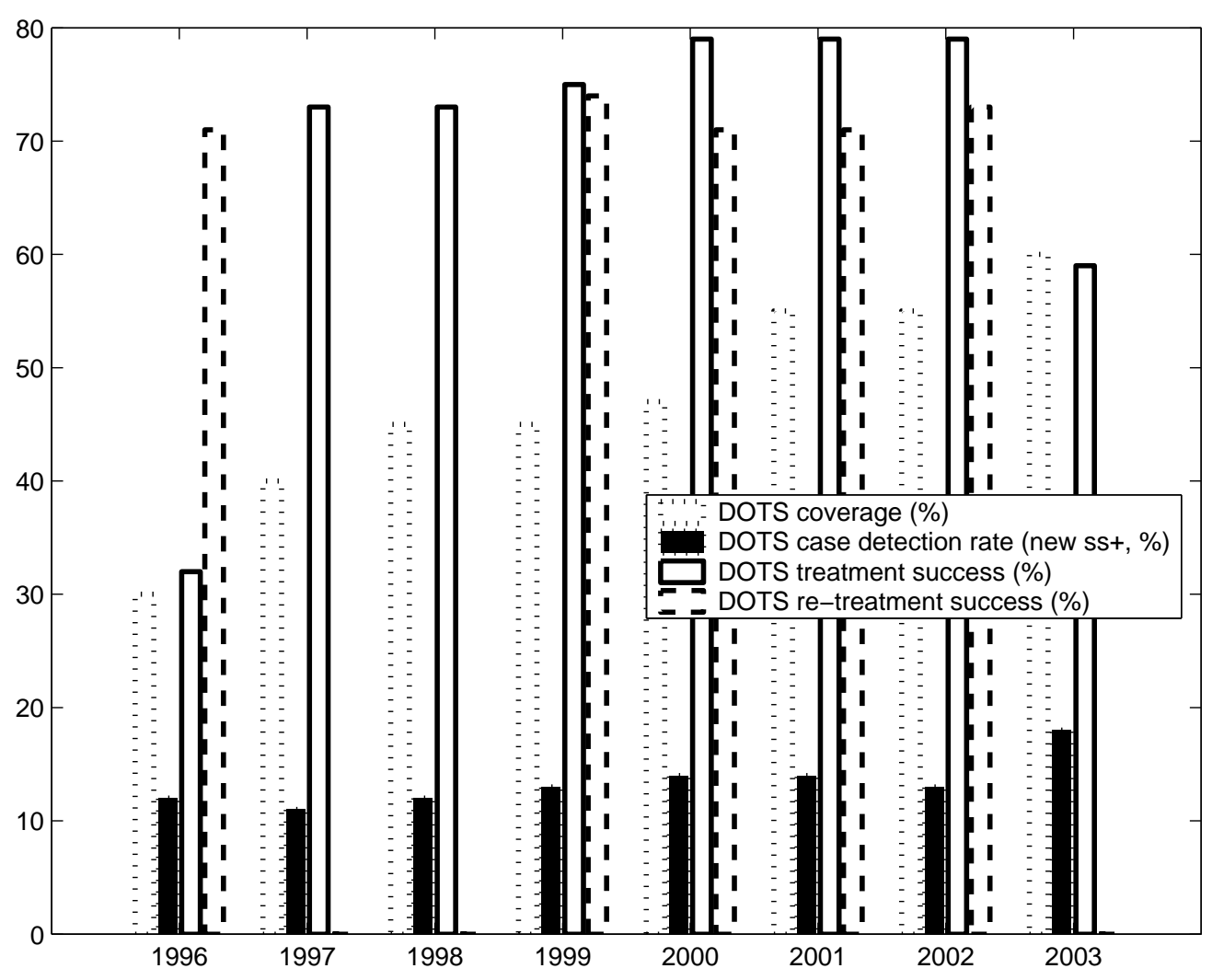

Figure 1: DOTS implementation in Nigeria (from [33]). 


\section{Tuberculosis Epidemiology}

TB was assumed to be on its way out in developed countries until the number of TB cases began to increase in the late 1980s [28]. The causes behind this recently observed increase of the number of active TB cases are the subject for many studies (see e.g., $[5,8,17])$. TB is an airborne transmitted disease. Mycobacterium tuberculosis droplets are released in the air by coughing or sneezing infectious individuals [28]. Tubercle bacillus contained in such droplets lives in the air for a short time (about two hours) and, therefore, it is believed that occasional contacts with TB-active persons (infectious individuals) rarely lead to transmission, and that the most secondary cases are the result of prolonged and sustained close contacts with a primary case. The case of a teacher-librarian with active TB who infected the children in her classroom but not the children who visited the library $[28,24]$ supports the assertion of the difference between the casual and the close contacts for there to be a transmission of the disease.

Latently infected individuals (inactive TB) become infectious (active TB) after a variable (typically long) latency period. Latent periods range from months to decades. Most infected individuals never progress towards the active TB state. On the other hand, an average infectious period is relatively short (a few months) and becomes shorter in developing nations due to the availability of treatment.

\section{Model Formulation}

We now proceed to formulation of a mathematical model that incorporates the detection and treatments under the DOTS program.

Typical TB treatment models are usually referred to as the SEIT (Susceptible - Exposed Infected - Treated) models (for example see [29, 32] and the survey article of Castillo-Chavez et al. [9]). To incorporate the DOTS implementation into this SEIT framework, we divided the infected class into two subclasses with different properties, namely the undetected infectious individuals and the detected infectious individuals. We will refer to such model a SEIJT (Susceptible - Exposed - Undetected Infected - Detected Infected - Treated) model.

Let $S(t), E(t), I(t), J(t), T(t)$ be the fractions of the susceptible individuals, the exposed (latent) individuals, the undetected and the detected infectious individuals and the treated/recovered individuals in the population, respectively, at time $t$. That is $N=S+E+I+J+T$ is the total population. Let $\Lambda$ be the recruitment rate into the susceptible class, which could include immigrants and/or newborns that are uninfected. We assume that $\mu$ is the per capita natural mortality rate; then $1 / \mu$ is an average lifespan of healthy individuals in the population. Let $d$ be the tuberculosis-induced mortality rate with $d_{1}$ being the rate for the undetected TB cases and $d_{2}$ being the rate for the detected TB cases, and let $\alpha_{1}, \alpha_{2}$ and $\gamma_{1}, \gamma_{2}$ be the per capita transmission rates for the susceptible class and the treated class, respectively. In this formulation, we also assume that the population is homogenous mixed, and all people are equally likely to be infected by the infectious individuals in a case of contact, and that transmission of the infection occurs with a bilinear incidence rate. 
Furthermore, TB exhibits strong dose-dependent effects (e.g., the above mentioned case of a teacher-librarian). To incorporate these into a model, we can assume that the length of the latent state inversely depends on the infective dose. In terms of a SEIJT model this means dependency on the number of the infectious individuals, and may be incorporated into a model in the form of the exogenous reinfection.

We assume that $\beta_{1}, \beta_{2}$ are the effective transmission rate for the latent class due to exogenous reinfection, and that $k$ is the rate of progression of the individuals in the latent state to active tuberculosis. We assume that $\omega$ is the fraction of those who are detected and treated under the DOTS program while the remaining $(1-\omega)$ is the fraction of the infectious individuals who are not detected. We assume that all patients under the DOTS (the detected infectious) complete their treatment. Let $r_{2}$ be the recovery/treatment rate for the detected infectious individuals under the DOTS scheme. The fraction of those detected cases who were successfully treated under the DOTS is $q$, while $p=1-q$ is the fraction of those whose treatment were unsuccessful and who thereafter join the latent class. The patients whose cases are not detected either die at the rate $d_{1}$, or self-cure and revert to latent state at the rate $r_{1}$ after an average of 2 years $[6,7,30]$. We also assume that the individuals in the latent class can be recovered/treated with a rate $r_{0}$.

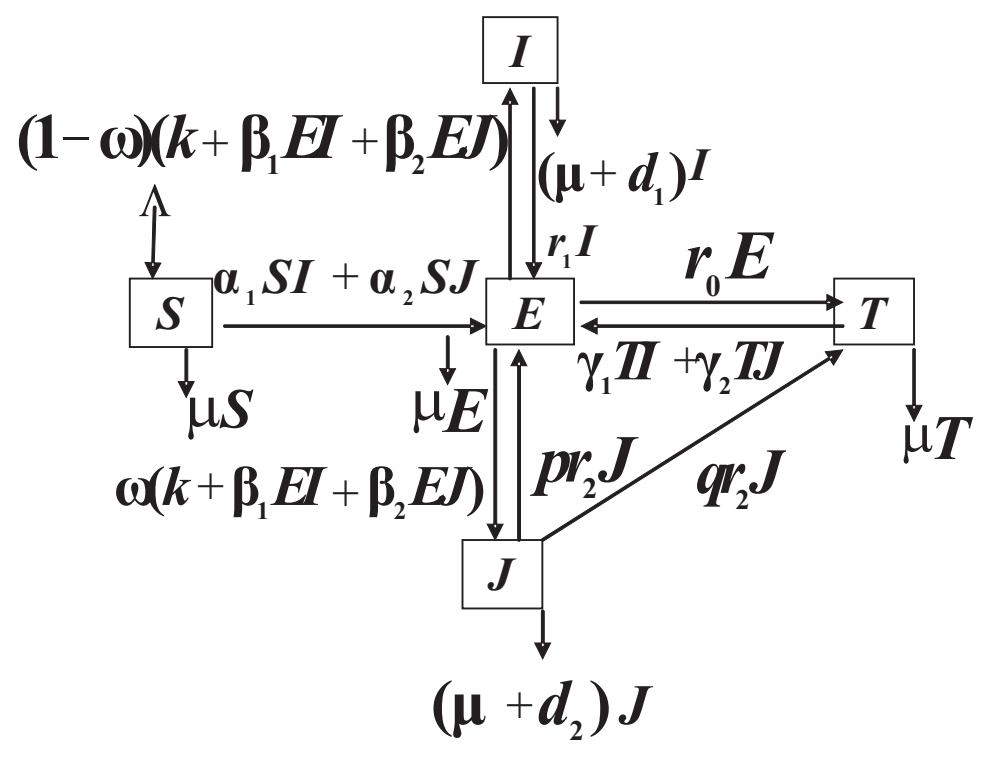

Figure 2: A general transfer diagram for the TB model.

Figure 2 shows the transfer diagram for a SEIJT model based on these assumptions. The above assumptions and the flow diagram lead to the following system of nonlinear ordinary differential 
equations:

$$
\begin{aligned}
\dot{S} & =\Lambda-\alpha_{1} S I-\alpha_{2} S J-\mu S, \\
\dot{E} & =\alpha_{1} S I+\alpha_{2} S J+\gamma_{1} T I+\gamma_{2} T J-\left(k+\mu+r_{0}+\beta_{1} I+\beta_{2} J\right) E+r_{1} I+p r_{2} J, \\
\dot{I} & =(1-\omega)\left(k+\beta_{1} I+\beta_{2} J\right) E-\left(\mu+d_{1}+r_{1}\right) I, \\
\dot{J} & =\omega\left(k+\beta_{1} I+\beta_{2} J\right) E-\left(\mu+d_{2}+r_{2}\right) J \\
\dot{T} & =r_{0} E+q r_{2} J-\gamma_{1} T I-\gamma_{2} T J-\mu T .
\end{aligned}
$$

For a SEIT model, it is usually assumed that the transmission rate for the treated is lower than that for the susceptibles, that is $0 \leq \gamma \leq \alpha$. For instance, for sexually transmitted diseases that may reflect an additional protection which can be a consequence of education. However, clinical observations give no evidence that treatment provides any additional protection against tuberculosis. That is, for TB the transmission coefficients for the susceptibles and the treated are equal or have negligible difference. However, if $\gamma_{i}=\alpha_{i}$, then the treated are equivalent to the susceptibles, and a SEIT model is equivalent to a SEIS model.

Furthermore, the nature of the exogenous reinfection is not clear in detail. For the sack of simplicity, we assume now that the dose-dependency directly affect the length of the latent state. We assume that a length of the latent period is $(a \cdot f(I, J))^{-1}$, where $a^{-1}>0$ is the average length of the latency when $I, J \rightarrow 0$, and $f(I, J)$ is a positive non-decreasing function such that $f(0,0)=1$. For simplicity we assume that $f(I, J)=1+\beta_{1} I+\beta_{2} J$.

The exposed individuals either are detected and enter $J$ class with the rate $\omega k\left(1+\beta_{1} I+\beta_{2} J\right)$, or are not detected and enter $I$ class with the rate $(1-\omega) k\left(1+\beta_{1} I+\beta_{2} J\right)$, or recover and return into the class of the susceptibles with the rate $r_{0}$; or they die. Therefore,

$$
k\left(1+\beta_{1} I+\beta_{2} J\right)+r_{0}<a\left(1+\beta_{1} I+\beta_{2} J\right) .
$$

With these assumption the SEIJT model (1) reduces to the following SEIJS model:

$$
\begin{aligned}
\dot{S} & =\Lambda-\alpha_{1} S I-\alpha_{2} S J-\mu S+q r_{2} J+r_{0} E, \\
\dot{E} & =\alpha_{1} S I+\alpha_{2} S J+r_{1} I+p r_{2} J-a\left(1+\beta_{1} I+\beta_{2} J\right) E, \\
\dot{I} & =(1-\omega) k\left(1+\beta_{1} I+\beta_{2} J\right) E-c I, \\
\dot{J} & =\omega k\left(1+\beta_{1} I+\beta_{2} J\right) E-b J .
\end{aligned}
$$

Here $b=\mu+d_{2}+r_{2}$ and $c=\mu+d_{1}+r_{1}$.

Further we will study the properties of this SEIJS model, referring to the SEIJT model (1) where this will be possible.

\section{Properties of the SEIJS model, and condition for eradication of tuberculosis}

It is easy to see that the model (3.3), as well as the model (1), always has a disease-free equilibrium state (DFE) $Q_{0}$ with the coordinates $S_{0}=\Lambda / \mu, E_{0}=I_{0}=J_{0}\left(=T_{0}\right)=0$. Apart from this equilibrium, both models can also have positive (endemic) equilibria. 
If a model has a positive equilibrium state and this equilibrium is stable, then all the subpopulations tends to the corresponding equilibrium levels. The disease will persist endemically in this case. If the disease-free equilibrium state is stable, then the population can remain diseasefree indefinitely (or as long as the conditions for the stability of DFE are valid). Moreover, if the disease-free equilibrium is asymptotically stable, then there is a critical level of the infectious population such that if the number of infectious individuals is below this critical level, then the disease cannot sustain in the population, and have to eventually disappear. The population will thereafter remain disease-free as long as the conditions for this stability are valid, even if the disease will be occasionally re-introduced into the population by migrants or travelers. The most favorable situation is, however, when the disease-free equilibrium in globally asymptotically stable; in this case the disease fades out from the population for any initial level of the infectious subpopulation.

The main goal of the DOTS is elimination of tuberculosis, or at least the reduction of the number of cases to an "acceptable" level. Therefore, in the terms of a mathematical model, the ultimate aim of DOTS is a situation when $(i)$ there is no stable positive equilibria, and when (ii) the disease-free equilibrium is stable.

The properties of these models, and existence of the endemic (positive) equilibria depends on the so-called basic reproduction number $R_{0}$, which is usually defined as the expected number of secondary cases produced, in a completely susceptible population, by a typical infective individual [14].

It is remarkable that the basic reproduction rates for the models (1) and (3.3) are the same. The basic reproduction number can be computed using the next generation matrix approach (see [32] for detail). Using this approach, we find that for the models (1) and (3.3) the basic reproduction number is

$$
R_{0}=R_{01}+R_{02}
$$

where $R_{01}=h Q_{1}$ and $R_{02}=h Q_{2}$, with

$$
h=\frac{k}{a-\frac{k(1-\omega) r_{1}}{c}-\frac{k p \omega r_{2}}{b}}
$$

and

$$
Q_{1}=(1-\omega) \frac{\alpha_{1} \Lambda}{\mu c}, \quad Q_{2}=\omega \frac{\alpha_{2} \Lambda}{\mu b} .
$$

It is noteworthy that $h$ is the fraction of the infected people who develop active TB.

The properties of DFE are completely defined by the following Theorem:

Theorem 4..1. If $R_{0} \leq 1$ for the model (3.3), then the infection-free equilibrium state $Q_{0}$ is the only non-negative equilibrium state of this model, and it is globally asymptotically stable.

Proof. 1. Firstly, we prove that the region

$$
\mathcal{D}=\left\{(S, E, I, J) \in \mathbb{R}_{\geq 0}^{4} \mid S+E+I+J \leq \Lambda / \mu\right\}
$$

is a positively invariant set and a global attractor of this system. That is, any phase trajectory initiated anywhere in the non-negative region $\mathbb{R}_{\geq 0}^{4}$ of the phase space eventually enters the region 
$\mathcal{D}$ and remains in $\mathcal{D}$ thereafter. We note that the non-negative region $\mathbb{R}_{\geq 0}^{4}$ is an invariant set of this system, and consider a Lyapunov function $W(S, E, I, J)=S+E+I+J$. Using (3.2), this function satisfies

$$
\begin{aligned}
\frac{d W}{d t} & =\frac{d}{d t}(S+E+I+J) \\
& =\Lambda-\mu S+r_{0} E-(a-k)\left(1+\beta_{1} I+\beta_{2} J\right) E-\left(\mu+d_{1}\right) I-\left(\mu+d_{2}\right) J \\
& <\Lambda-\mu S-\left(\mu+d_{1}\right) I-\left(\mu+d_{2}\right) J .
\end{aligned}
$$

That is, $\frac{d W}{d t}<-d_{1} I-d_{2} J<0$ for all $(S, E, I, J) \in\left\{\mathbb{R}_{\geq 0}^{4} \backslash \mathcal{D}\right\}$, and hence, by the virtue of Lyapunov-LaSalle asymptotic stability theorem [19], the set $\mathcal{D}$ is a global attractor and an invariant set of this system.

2. We note that the system has no equilibria outside of the region $\mathcal{D}$. Indeed, the derivative of a Lyapunov function must be equal to zero at an equilibrium state, whereas $\frac{d W}{d t}<0$ for all $(S, E, I, J) \in\left\{\mathbb{R}_{\geq 0}^{4} \backslash \mathcal{D}\right\}$.

3. Now we have to prove the Theorem in the region $\mathcal{D}$. Let consider a Lyapunov function

$$
U(S, E, I, J)=S-S_{0} \ln S+E+B I+C J,
$$

where $B=\frac{1}{c}\left(\alpha_{1} \frac{\lambda}{\mu}+r_{1}\right)$ and $C=\frac{1}{b}\left(\alpha_{2} \frac{\lambda}{\mu}+p r_{2}\right)$, with $b$ and $c$ as above. Recalling that $S_{0}=$ $\Lambda / \mu$ and $p+q=1$, this function satisfies

$$
\begin{aligned}
\frac{d U}{d t}= & \Lambda-\alpha_{1} S I-\alpha_{2} S J-\mu S+q r_{2} J+r_{0} E-\Lambda \frac{S_{0}}{S} \\
& +\alpha_{1} S_{0} I+\alpha_{2} S_{0} J+\mu S_{0}-q r_{2} J \frac{S_{0}}{S}-r_{0} E \frac{S_{0}}{S} \\
& +\alpha_{1} S I+\alpha_{2} S J+r_{1} I+p r_{2} J-a\left(1+\beta_{1} I+\beta_{2} J\right) E \\
& +B\left((1-\omega) k\left(1+\beta_{1} I+\beta_{2} J\right) E-\left(\mu+d_{1}+r_{1}\right) I\right) \\
& +C\left(\omega k\left(1+\beta_{1} I+\beta_{2} J\right) E-\left(\mu+d_{2}+r_{2}\right) J\right) \\
= & \Lambda\left(2-\frac{S_{0}}{S}-\frac{S}{S_{0}}\right)+q r_{2} J\left(1-\frac{S_{0}}{S}\right)+r_{0} E\left(1-\frac{S_{0}}{S}\right) \\
& +\left(\alpha_{1} S_{0}+r_{1}-B c\right) I+\left(\alpha_{2} S_{0}+p r_{2}-C b\right) J \\
& +((1-\omega) B k+\omega C k-a)\left(1+\beta_{1} I+\beta_{2} J\right) E .
\end{aligned}
$$

Here, $2-\frac{S_{0}}{S}-\frac{S}{S_{0}}=\left(1-\frac{S_{0}}{S}\right)\left(1-\frac{S}{S_{0}}\right) \leq 0$ for all $S \geq 0$. Furthermore, $\alpha_{1} S_{0}+r_{1}=B c$, $\alpha_{2} S_{0}+r_{2}=C b$, and

$$
\begin{aligned}
(1-\omega) B k+\omega C k-a & =(1-\omega) \frac{\alpha_{1}}{c} \frac{\Lambda}{\mu} k+\omega \frac{\alpha_{2}}{b} \frac{\Lambda}{\mu} k-\left(a-(1-\omega) k \frac{r_{1}}{c}-\omega k \frac{p r_{2}}{b}\right) \\
& =\frac{1}{h}\left(Q_{1} h+Q_{2} h-1\right)=\frac{1}{h}\left(R_{0}-1\right) .
\end{aligned}
$$

That is, $R_{0} \leq 1$ ensures that $\frac{d U}{d t} \leq 0$ holds for all $(S, E, I, J) \in \mathcal{D}$, where the equality $\frac{d U}{d t}=0$ holds only when $S=S_{0}$. In the set $\mathcal{D}$, the equality $S=S_{0}$ holds only on the straight line 
$S=S_{0}, I=J=0$; it is easy to see that this line is not an invariant set of this system, and that there is no equilibrium states of the system on this line. Therefore, by Lyapunov-LaSalle asymptotic stability theorem [19], $R_{0} \leq 1$ ensures that the disease-free equilibrium state $Q_{0}$ is globally asymptotically stable in $\mathbb{R}_{\geq 0}^{4}$.

4. The uniqueness of the equilibrium state $Q_{0}$ in $\mathbb{R}_{\geq 0}^{4}$ follows from the fact that $Q_{0}$ is the only point in $\mathbb{R}_{\geq 0}^{4}$ where the derivatives of both Lyapunov functions, namely $W$ and $U$, are equal to zero.

This completes the proof.

We have to remark that $R_{0} \leq 1$ ensures that $\frac{d U}{d t}<0$ holds everywhere in $\mathbb{R}_{\geq 0}^{4}$, apart from the three-dimensional space $S=S_{0}$, where $\frac{d U}{d t}=0$. By Lyapunov-LaSalle asymptotic stability theorem, this implies that the largest invariant set of the system, which is entirely contained in the 3-dimensional space $S=S_{0}$, is globally asymptotically stable. However, proving that the system has no invariant sets in the space $S=S_{0}$ is a non-trivial problem, and it is simpler, therefore, to introduce an auxiliary Lyapunov function $W$ and prove that there is no invariant sets outside of the region $\mathcal{D}$.

The global stability of the disease-free equilibrium state implies that for any initial level of infection the disease will eventually fade out from the population if $R_{0} \leq 1$ holds. This gives us the condition for the detection rate $\omega$ that is necessary for elimination of the disease. It is easy to see that $R_{0}<1$ holds if

$$
\omega \geq \omega_{c}=\frac{\frac{\alpha_{1} \Lambda / \mu+r_{1}}{c}-\frac{a}{k}}{\frac{\alpha_{1} \Lambda / \mu+r_{1}}{c}-\frac{\alpha_{2} \Lambda / \mu+p r_{2}}{b}},
$$

where $c=\mu+d_{1}+r_{1}$ and $b=\mu+d_{2}+r_{2}$.

$R_{0}>1$ implies that a single infected individual introduced into entirely susceptible population will produce an average more that one infected in the next generation; that is in this case the disease may invade and persists in a population. Standard linear analysis shows that when the basic reproduction rate $R_{0}$ is greater than 1 , then DFE is unstable. That is a supercritical bifurcation occurs at $R_{0}=1$, and a stable positive equilibrium appears.

\section{Parameter estimation and numerical results}

We now give estimations for the parameters of the model.

We set a year as an unit of time. The natural mortality $\mu$ is postulated to be equal to the inverse of the life expectancy at birth, which is now about 49 years in Nigeria [31]; that is $\mu=1 / 49=$ $0.02041 \mathrm{yr}^{-1}$. The recruitment rate $\Lambda$ controls the total population size because $N \sim \Lambda / \mu$. We set $\Lambda=\mu \times 10^{5} \mathrm{yr}^{-1}[28]$.

Per capita TB-induced mortality rate varies from country to country. That is around $0.07 \mathrm{yr}^{-1}$ in developed countries, but could be as high as $0.395 y^{-1}$ in some African countries [1, 27]. An intermediate value of $0.1 \mathrm{yr}^{-1}$ can be applied to most developed and developing countries [1]. For this simulation we set the TB-induced mortality rate for the detected infectious class to $0.395 \mathrm{yr}^{-1}$. The infected who are not detected either die (at the rate $d_{1}$ ), or self-cure (at the rate $r_{1}$ ) after an average infectious period of 2 years [6]; hence, following [6, 15, 31], we set $d_{1}=0.3 y^{-1}$. 
We take the recovery rates as the inversion of an average period between the TB activation and the moment of recovery. We assume that the cases are either detected within 4 months after an infection, or are not detected at all [6]. According to Styblo et al. [30], the time interval between TB activation and recovery by treatment is between 4 and 6 months, while in [16] the period of treatment is about 6 to 9 months. Either of these implies that $r_{2}>1$. If we take the treatment period to be equal to 6 months, following [29], we have that the recovery rate $r_{2}=2$ per personyear. This may be compared with the recovery rate $1 / 0.67=1.5$ per person-year that was used in [30].

We will set $r_{0}$ equal to 1.5 per person-year. As it was mentioned earlier, the individuals with active TB who are not detected after an average of 2 years either die (with the rate $d_{1}=0.3$ ), or self-cure. Self-cure in this case refers to the active cases which revert to the latent infection state without being treated. Assuming that an average undetected infectious period is equal to 2 years and that $d_{1}=0.3$, Dye et al. [15] obtained the self-cure rate $r_{1}=0.2$ per person-year; we will use this figure.

The steady rise of the number of the notified cases of TB was observed since 1995 in Nigeria [33]. For the fifteen years the TB-induced mortality has quadrupled, from 24,461 death in 1990 to 99,938 death recorded in 2005 [33]. Since 1997, the DOTS treatment success rate $q$ has stabilized at about $75 \%$ of all detected cases, while the estimation for the case detection rate has ranged from $11 \%$ to $21 \%$ in 2004 [33]. This figure is insufficient compared with the WHO target for high-burden countries like Nigeria, which is $70 \%$ case detection rate and at least $85 \%$ successful cure rate.

The fraction of the infected people who develop active TB,

$$
h=\frac{k}{\left(k+\mu+r_{0}\right)-\frac{k(1-\omega) r_{1}}{c}-\frac{k p \omega r_{2}}{b}},
$$

has been estimated to be between $5 \%$ and $10 \%$ in developed countries, while for developing countries this could be about $15 \%$, and in some extreme cases could reach $30 \%$ [28]. For this study we set $h=0.15$. Hence an average per capita rate of progression to active TB is

$$
k=\frac{h \times\left(\mu+r_{0}\right)}{1-h \times\left(1-\frac{r_{1}(1-\omega)}{c}-\frac{p \omega_{2} r_{2}}{b}\right)} .
$$

For the past eight years $\omega$ was ranging from 0.11 to 0.21 in Nigeria (the last value is the current situation). With $h=0.15$, we observe that this variation of $\omega$ does not make much difference in the progression rate: for these values of $\omega, k$ ranges from 0.2517 (for $\omega=0.11$ ) to 0.2527 (for $\omega=0.21$ ). Even with $h=0.1$ (which is hardly feasible for a developing country), the progression rate does not change significantly. Hence we can assume that the progression rate has been near constant over the last decade in Nigeria.

Estimating the transmission parameters $\alpha_{1}$ and $\alpha_{2}$ is the most difficult task. Usually these parameters are estimated using a known value of the basic reproduction number $R_{0}$. The transmission coefficients can be chosen by matching them with $Q_{1}, Q_{2}$ and the per capita number of the secondary infections produced by the active TB cases $Q=Q_{1}+Q_{2}$. Then, using

$$
Q_{1}=\frac{(1-\omega) \alpha_{1} \Lambda / \mu}{c}, \quad Q_{2}=\frac{\omega \alpha_{2} \Lambda / \mu}{b},
$$


we have $\alpha_{1}=\Lambda c \times Q_{1} /(1-\omega) \mu$ and $\alpha_{2}=\Lambda b \times Q_{2} / \omega \mu$, where $0<\omega<1$. Table 1 summarises the results for several scenarios corresponding to different values of $Q$. The effect of variation of the detection rate $\omega$ on the basic reproduction number can be seen from this Table. Apart from the transmission parameters, all the other parameters for this model were adapted from reliable demographic and epidemiological data on TB and DOTS implementation in Nigeria; the transmission coefficients are the only parameters with a significant degree of uncertainty. For the exogenous re-infection parameters we set $\beta_{1}=\alpha_{1}$ and $\beta_{2}=\alpha_{2}$.

\begin{tabular}{|c|c|c|}
\hline$Q$ & $\omega$ & $R_{0}$ \\
\hline 10 & 0.62 & 1.5 \\
9.7 & 0.72 & 1.455 \\
8.9 & 0.42 & 1.335 \\
8.8 & 0.39 & 1.32 \\
7 & 0.24 & 1.05 \\
6.7 & 0.22 & 1.005 \\
\hline
\end{tabular}

Table 1: Relationship between the fraction of the detected patients under DOTS, the number of secondary infections and the basic reproduction number.

Figure 3 shows the results of the numerical simulation for the model with the mentioned parameter values. This simulation shows the increasing number of the undetected cases, and the effect of this on the fraction of the latent infections. This simulation demonstrates an initial drop in the number of latent infections and detected infectious cases, which is followed by a gradual increase. This qualitative picture coincides with the existing clinical data that exhibit the increasing number of incidence in Nigeria in the last 10 years [33]. It appears that the high treatment rate for the latent and detected infectious classes accounts for the initial drop in both these classes. However, the continued presence of the huge pool of the undetected cases may likely have triggered the sudden rise in the number of latent infections and active TB cases in Nigeria.

\section{Discussion and Conclusions}

According to clinical data, in Nigeria currently the incidence of tuberculosis is on the increase [33]. Among other factors, that may be attributed to the failure to detect a huge number of active TB cases, which are primarily responsible for the spread of the infection. In order to control the incidence of tuberculosis, this issue must be addressed.

In this research, we have examined the effects of DOTS on the dynamics of tuberculosis vis a vis the fraction of the active cases detected. To explore the effect of DOTS, we formulate a mathematical model that incorporates the fraction of the detected cases undergoing treatment under DOTS and the other fraction that are not detected. The qualitative analysis of this model shows that there is a disease free equilibrium state, and that this equilibrium state is globally asymptotically stable provided that the fraction of detected cases exceeded a certain critical value $\omega_{c}$. This implies that the disease will eventually disappear if this critical level of detection will be reached. The 

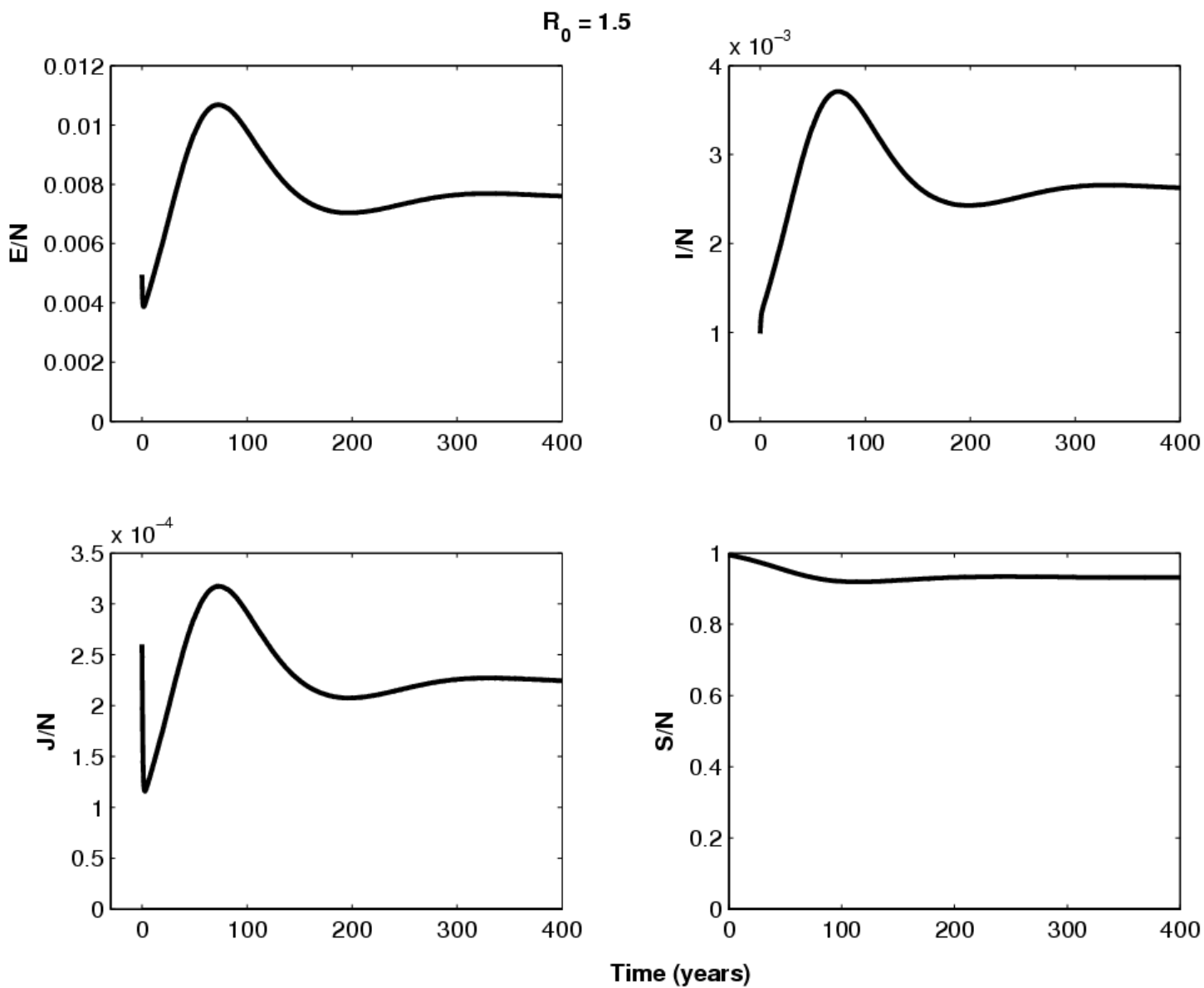

Figure 3: Population fractions for $Q=10$. 
obvious requirement for minimization of the incidence of tuberculosis is also increasing the detection rate. The results of numerical simulations support these conclusions: analysis and simulations show that the role of the case detection is crucial for reducing the incidence of TB. Increasing the probability of detection reduces the basic reproduction number, and hence the number of new infection; the critical detection level corresponds to $R_{0}=1$. The basic reproduction number $R_{0}$ is one of the basic concept of epidemiology, which usually determines behaviour of a disease in a given population. If the critical level of the detection rate is not reached, then $R_{0}>1$, and the disease will persist in the population endemically.

The obvious conclusion is that the improvement of the case detection must be on the list of priorities. Expansion of DOTS should not just concentrate on the improvement of the treatment alone, but emphases should be placed on detection as well. At the first instance, the dynamic nature of the programme should be used to introduce screening of close contacts of the detected cases to find out how many of these have latent or active tuberculosis. The approach of screening household contacts used by Becerra et al. [3] may be adopted for Nigeria. The objective of the study of Becerra et al. [3] was to find a better strategy for a high-incidence population in northern Lima, Peru. The results show that the tuberculosis prevalence detected through combined active and passive case detection among 1,094 household contacts was $0.91 \%$, compared with $0.18 \%$ of cases detected through passive case finding alone. This implies that the risk of infection for the household contacts of a symptomatic case is very high, and that contact tracing may be a simple and inexpensive option for improving the case detection rates.

The current rate of $21 \%$ case detection in Nigeria is insufficient and in fact jeopardises all the efforts of the DOTS program. The impact of the enormous number of undetected case is not only seen in an increase in the incidence of active TB; there also is a large pool of the latent individuals, which is hardly surprising since the majority of secondary infections is caused by the undetected individuals. In fact, depending on the actual contact rate, bringing down the incidence of tuberculosis can require the case detection rate a little higher than $70 \%$. This corresponds with the WHO recommendations and with figures in Table 1.

The results of the simulations suggest that despite the improvement in tuberculosis treatment success rate achieved under DOTS in Nigeria, the high number of undetected cases remains a major obstacle preventing the long-term success of DOTS in TB control. Current WHO policy emphasizes passive case finding in contrast with the identification of cases through screening [3, 25]. This strategy has been based on the expectation that passive detection of individuals, who is ill enough to seek medical attention, is far more cost-effective than population-based screening, and that the compliance will be higher in those who have identified themselves as symptomatic [3]. However, the failure of national tuberculosis programs to detect the vast majority of new infectious cases suggests that active screening strategies should be reevaluated in an attempt to improve case detection and, thereby, to increase access to TB treatment [21]. Therefore, if DOTS has to be expanded, that should at least include screening of close contacts of the treated individuals. Subsequently, these should be placed under the DOTS scheme. Invariably, this will reduce the number of both the latent and the infectious individuals. DOTS must go beyond treatment; this programme should entail case detection as its essential part as well. DOTS in Nigeria and in developing countries in general will only make sense if the current treatment rate for detected infectious individuals 
is sustained, or even improved upon, and if a strategy targeted to increasing the case detection rate is used. Once an infectious person is detected, all close associates, such as family members, should be screened as well. Since the treatment success rate is very impressive in Nigeria, with the detection level $\omega_{c}$ achieved, reducing the value of $R_{0}$ below one is possible.

Presently, we have to state that despite all efforts, DOTS has not succeeded in reduction of the incidence level of tuberculosis in Nigeria. We believe that the reason for this is that only a small fraction of the infected individuals are actually treated under DOTS, whereas the vast majority of the infected are undetected and actually causes an increase in the number of new cases. Therefore, the obvious conclusion is that DOTS service in Nigeria should be expanded to take care of case detection, as this will assist in reducing the burden of tuberculosis on the Nigerian economy and the community in general.

We also suggest that expanding DOTS onto the rural areas will be necessary. This have to include providing transportation for the patients to receive their medication and if possible screening of the families of these patients. Medical personnel can be paid to go to these areas to provide medication to the patients as well as to simultaneously conduct screening for the patients' contacts. At this instance, we do not consider the economic implication of adoption the findings. We have merely provided the condition for the case detection level for DOTS implementation that leads to reduction of the incidence of tuberculosis in Nigeria, or even it elimination.

DOTS as a strategy adopted by WHO, which is targeted to reduce the number of active tuberculosis cases as well as promote the proper treatment of the patients. The insights gained from analysis of the mathematical model formulated in this article can be useful in the study of the impact of DOTS and its relationship with case detection on the dynamics of tuberculosis in Nigeria or any other country. This may assist health and government authorities to plan improvement for the case detection which will help in bringing down the incidence and prevalence of TB in the country.

\section{References}

[1] J.P. Aparicio, A.F. Cappuro, C. Castillo-Chavez. Transmission and dynamics of tuberculosis on generalized households. J. Theor. Biol., 206 (2000) 327-341.

[2] J.P. Aparicio, J.C. Hernandez. Preventive treatment of tuberculosis through contact tracing. Mathematical studies on human disease dynamics: emerging paradigms and challenges. AMS Contemporary Mathematics Series, Vol. 410. Abba B. Gumel (Editor-in-Chief), Carlos Castillo-Chavez (Ed.), Ronald E. Mickens (Ed.), Dominic P. Clemence(Ed.), 2006.

[3] M.C. Becerra, I.F. Pachao-Torreblanca, J. Bayona, R. Celi, S.S. Shin, J.Y. Kim, P.E. Farmer, M. Murray. Expanding tuberculosis case detection by screening household contacts. Public Health Reports, 120, 2005.

[4] W.R. Bishai, N.M. Graham, S. Harrington, D.S. Pope, N. Hooper, J. Astemborski, L. Sheely, D. Vlahov, G.E. Glass, R.E. Chaisson. Molecular and geographic patterns of tuberculosis transmission after 15 years of directly observed therapy. J. Am. Med. Assoc., 280 (1998), 1679-1684. 
[5] S.M. Blower, P.M. Small, P.C. Hopwell. Control strategies for tuberculosis epidemics: new models for old problems. Science, 273 (1996) 497.

[6] M.W. Borgdorff. New measurable indicator for tuberculosis case detection. Emerging Infectious Diseases, 10 (2004), No. 9.

[7] M.W. Borgdorff, K. Floyd, J.F. Broekmans. Interventions to reduce tuberculosis mortality and transmission in low and middle-income countries. Bull World Health Organ, 80 (2002), $17-227$.

[8] C. Castillo-Chavez, Z. Feng. To Treat or Not to Treat: the Case of Tuberculosis. J. Math. Biol., 35 (1997), 629.

[9] C. Castillo-Chavez, B. Song. Dynamical models of tuberculosis and their applications. Mathematical Biosciences and Engineering, 1 (2994), No 2, 361-404.

[10] E.D. Chan, M.D. Iseman. Current medical treatment for tuberculosis. B.M.J., 325 (2992), 1282-1286.

[11] C.P. Chaulk, M. Friedman, R. Dunning. Modeling the epidemiology and economics of directly observed therapy in Baltimore. Int. J. Tuberc. Lung. Dis., 4 (2000) 201-207.

[12] C.P. Chaulk, V.A. Kazandjian. Directly observed therapy for treatment completion of pulmonary tuberculosis: consensus statement of the Public Health Tuberculosis Guidelines Panel. J.A.M.A., 279 (1998), 943-948.

[13] D.L. Cohn, B.J. Catlin, K.L. Peterson, F.N. Judson, J.A. Sbarbaro. A 62-dose, 6-month therapy for pulmonary and extrapulmonary tuberculosis: A twice-weekly, directly observed, and cost-effective regimen. Ann. Intern. Med., 112 (1990), 407-415.

[14] O. Diekmann, J.A. Heesterbeek, J.A.J. Metz. On the definition and the computation of the basic reproductive ratio, $R 0$ in models of infectious diseases in heterogeneous populations. J. Math. Biol., 28 (1990), 365-382.

[15] C. Dye, G.P. Garnett, K. Sleeman, B.G. Williams. Prospects for worldwide tuberculosis control under the WHO DOTS strategy. Directly observed short-course therapy. Lancet, 352 (1998), 1886-1891.

[16] W. Feng, Z.and Huang, C. Castillo-Chavez. On the role of variable latent periods in mathematical models for tuberculosis. J. Dynamics and Differential Equations, 13 (2991), 425452.

[17] Z. Feng, C. Castillo-Chavez, A.F. Capurro. A model for tuberculosis with exogenous reinfection. Theor. Pop. Biol., 57 (200), 235.

[18] H.W. Hethcote. The mathematics of infectious diseases. SIAM Review, 42 (2000), No. 4, 599-653. 
[19] J. La Salle, S. Lefschetz. Stability by Liapunov's direct method. Academic Press, New York, 1961.

[20] G. Magombedze, W. Garira, E. Mwenje. Modelling the human immune response mechanisms to mycobacterium tuberculosis infection in the lungs. Mathematical Biosciences and Engineering, 3, (2006), No 4, 661-682.

[21] C.J. Murray, J.A. Salomon. Expanding the WHO tuberculosis control strategy: rethinking the role of active case-finding. Int. J. Tuberc. Lung. Dis., 2 (1998), No. 9 Suppl 1, 9-15.

[22] R.J. North, J. Yu-Jin. Immunity to tuberculosis. Annu. Rev. Immunol., 22 (2004), 599-623.

[23] The Punch. Nigeria ranks fourth among TB high-burden countries. The Punch Newspaper, page 3, January 15, 2007.

[24] J. Raffalii, K.A. Sepkowitz, D. Armstrong. Community-based outbreaks of tuberculosis. Arch. Int. Med., 156 (1996), 1053.

[25] M.C. Raviglione, A. Pio. Evolution of WHO policies for tuberculosis control, 1948-2001. Lancet, 359 (2002), 775-80.

[26] N.W. Schluger, Rom W.N. The host immune response to tuberculosis. Am. J. Respir. Crit. Care. Med., 157 (1998), 679-691.

[27] D.E. Snider, M. Raviglione, A. Kochi. Epidemiology of tuberculosis. In: B. Bloom, Editor, Global Burden of Tuberculosis, Tuberculosis: Pathogenesis, Protection and Control, ASM Press, Washington, D.C., 1994.

[28] B. Song, C. Castillo-Chavez, J.P. Aparicio. Tuberculosis models with fast and slow dynamics: the role of close and casual contacts. Math. Biosci., 180 (2002), 187-205.

[29] A. Ssematimba, J.Y.T. Mugisha, L.S. Luboobi. Mathematical models for the dynamics of tuberculosis in density-dependent populations: the case of internally displaced peoples camps (IDPCs) in Uganda. Journal of Mathematics and Statistics, 1 (2005), No. 3, 217-224.

[30] K. Styblo, J.R. Bumgarner. Tuberculosis can be controlled with existing technologies: evidence. The Hague: Tuberculosis Surveillance Research Unit, pages 60-72, 1991.

[31] UNAIDS-WHO. UNAIDS/WHO Epidemiological Fact Sheet. www.unaids.org, 2004.

[32] P. van den Driessche, J. Watmough. Reproduction numbers and sub-threshold endemic equilibria for compartmental models of disease transmission. Math. Biosci., 180 (2002), 29-48.

[33] WHO. Global Tuberculosis Control. WHO Report, Geneva, 2006. 